\title{
The role of omental biopsy in endometrial cancer staging
}

\author{
M. Abu Freij • Nikolaos Burbos • D. Mukhopadhyay • \\ R. Lonsdale $\cdot$ S. Crocker $\cdot$ J. J. Nieto
}

Received: 31 January 2009 /Accepted: 27 February 2009/Published online: 19 March 2009

(C) Springer-Verlag 2009

\begin{abstract}
Omental biopsy is not part of FIGO staging for endometrial cancer. The few studies that have looked into this matter have had conflicting results. This is the largest study in terms of the number of cases studying the incidence of omental involvement in endometrioid and non-endometrioid endometrial cancer. A retrospective study assessing 248 cases of endometrial cancer with omental biopsy at the time of primary surgical treatment for endometrial cancer at the Gynaecological Oncology Centre, Norfolk and Norwich University Hospital between January 2004 and May 2008. Demographic, clinico-pathologic and surveillance data were collected from hospital records, operative notes and histopathology results. The histology included tumour type, stage, grade and omental biopsy. All histological types were included in the study. Two hundred and forty eight patients had an omental biopsy at the time of primary surgical treatment for endometrial cancer during the study period. Of them, 187 cases were stage I, 27 stage II, 27 stage III and seven stage IV. According to histological type, $202(81.4 \%)$ had endometrioid, 20 $(8.0 \%)$ serous papillary, $20(8.0 \%)$ malignant mixed
\end{abstract}

M. A. Freij $(\bowtie) \cdot$ S. Crocker $\cdot$ J. J. Nieto

Gynaecological Oncology,

Norfolk and Norwich University Hospital,

Colney Lane,

Norwich, Norfolk NR4 7UY, UK

e-mail: Mazen2K@yahoo.com

N. Burbos · D. Mukhopadhyay

Obstetrics and Gynaecology,

Norfolk and Norwich University Hospital,

Norfolk, UK

R. Lonsdale

Pathology, Norfolk and Norwich University Hospital,

Norfolk, UK
Mullerian tumour (MMMT), three (1.2\%) clear cell and three (1.2\%) sarcoma. Overall, six cases $(2.4 \%)$ had omental involvement, 4/202 (1.98\%) with endometrioid type, $1 / 20(5.0 \%)$ with serous papillary type and $1 / 20$ (5.0\%) MMMT. Eighty four percent of omental metastases (five cases) were macroscopic and noted at operation. The overall risk of omental metastases is $2.4 \%$ and in the absence of gross lesions the risk is around $0.4 \%$. Most omental metastases can be diagnosed by careful inspection and palpation of the omentum. The possibility of missing microscopic disease is low. Based on these figures and possible increase in morbidity and operative time, omental biopsy cannot be justified as a standard procedure in endometrial cancer staging.

Keywords Omental biopsy - Endometrial cancer

\section{Introduction}

Uterine cancer is the second most common gynaecological malignancy and the fifth most common cancer in women in the UK with around 6,400 cases diagnosed each year [1]. Seventy five percent of patients survive for 5 years, the majority is diagnosed in an early stage and most present with postmenopausal bleeding. Worldwide, the number of deaths from endometrial cancer has increased significantly since 1987 [2]. Endometrial cancer metastasizes by direct spread into the myometrium, extending to the cervix [3], haematogenous dissemination, lymphatic embolisation and peritoneal seeding [4]. Endometrial cancer can metastasize to the omentum through peritoneal seeding by-passing the myometrium and regional lymphatics.

FIGO adopted surgico-pathologic staging for endometrial cancer in 1988. The standard staging involves total abdominal 
Table 1 Patients who had an omental biopsy at the time of surgical staging for endometrial cancer

\begin{tabular}{lll}
\hline & Number & Percent \\
\hline Age (33-93) & 12 & \\
$<50$ & 236 & 06 \\
$\geq 50$ & & 94 \\
Grade & 44 & \\
1 & 100 & 19 \\
2 & 104 & 40 \\
3 & & 41 \\
Stage & 33 & \\
Ia & 103 & 15 \\
Ib & 51 & 40 \\
Ic & 27 & 20 \\
II & 27 & 11 \\
III & 7 & 11 \\
IV & & 03 \\
Histological type & 202 & \\
Endometrioid & 20 & 81.4 \\
Serous papillary & 20 & 8.1 \\
MMMT & 3 & 8.1 \\
Clear cell & 3 & 1.2 \\
Sarcoma & & 1.2 \\
\hline
\end{tabular}

hysterectomy and bilateral salpingo-oophorectomy, evaluation of pelvic and para-aortic lymph nodes, peritoneal washings, palpation and inspection of the abdominal organs in attempt to identify extrauterine spread [5]. Omental biopsy is not part of FIGO staging for endometrial cancer.

In the UK, lymphadenectomy is not done in all cases of endometrial cancer and the role of lymphadenectomy remains debatable. We thought omental biopsy may improve staging; detection of occult omental metastases will upstage the cancer to stage IVb.

Previous studies including by one of the current authors had suggested that the omentum might be involved in up to $10 \%$ of the cases [6-10]. Gehrig et al. [11] suggested that most omental metastases can be diagnosed by gross
Table 3 Recent studies looking at omental biopsy in endometrial cancer

\begin{tabular}{llll}
\hline Study & $\begin{array}{l}\text { Number } \\
\text { of cases }\end{array}$ & $\begin{array}{l}\text { Omental } \\
\text { involvement } \\
\text { cases (\%) }\end{array}$ & $\begin{array}{l}\text { Number of } \\
\text { cases with } \\
\text { microscopic } \\
\text { metastasis }\end{array}$ \\
\hline Present study & 248 & $6(2.4)$ & $1 / 6$ \\
Saygili et al. [6] & 97 & $6(6.0)$ & $4 / 6$ \\
Chen SS et al. [7] & 84 & $7(8.3)$ & $5 / 7$ \\
Nieto et al. [8] & 80 & $6(7.5)$ & $2 / 6$ \\
Dilek et al. [9] & 51 & $3(6.0)$ & $3 / 3$ \\
Fujiwara et al. [10] & 134 & $4(3.0)$ & $1 / 4$ \\
\hline
\end{tabular}

visualisation of the omentum. The sensitivity of a visually negative omentum is 0.89 . It remains uncertain whether omental biopsy should be part of routine staging of endometrial cancer. So far, few studies have looked into this and have had conflicting results. Up to date, this is the largest study in terms of the number of cases studying the incidence of omental involvement in endometrioid and nonendometrioid endometrial cancer.

\section{Materials and methods}

A retrospective study assessing 248 cases of endometrial cancer having omental biopsy at the time of primary surgical treatment for endometrial cancer at the Gynaecological Oncology Centre, Norfolk and Norwich University Hospital between January 2004 and May 2008 was conducted. Demographic, clinico-pathologic and surveillance data were collected from hospital records, operative notes and histopathology results. The histology included tumour type, stage, grade and omental biopsy. All histological types were included in the study.

The omentum was inspected and infracolic omentectomy was performed in the presence of gross lesions. Otherwise, a representative sample of the omentum was obtained. The relationship between grade, depth of myometrial invasion,

Table 2 Clinical features of six patients with omental involvement

\begin{tabular}{|c|c|c|c|c|c|c|c|}
\hline Case & Cell type & Grade & $\begin{array}{l}\text { Myometrial } \\
\text { invasion }\end{array}$ & $\begin{array}{l}\text { Adnexal } \\
\text { involvement }\end{array}$ & $\begin{array}{l}\text { Peritoneal } \\
\text { washings }\end{array}$ & $\begin{array}{l}\text { Omental } \\
\text { metastasis }\end{array}$ & $\begin{array}{l}\text { Follow-up (month) } \\
\text { to June } 08\end{array}$ \\
\hline 1 & Endometrioid & 3 & $>50 \%$ & Positive & Positive & Macromets & Death (7) \\
\hline 2 & Endometrioid & 2 & $<50 \%$ & Negative & Negative & Micromets & Alive (18) \\
\hline 3 & Endometrioid & 3 & $>50 \%$ & Negative & Positive & Macromets & Alive (18) \\
\hline 4 & Endometrioid & 2 & $>50 \%$ & Negative & Positive & Macromets & Alive (12) \\
\hline 5 & Serous papillary & 3 & $>50 \%$ & Positive & Positive & Macromets & Death (1) \\
\hline 6 & MMMT & 3 & $>50 \%$ & Positive & Positive & Macromets & Death (3) \\
\hline
\end{tabular}


histological type, peritoneal cytology and omental metastases was assessed.

\section{Results}

Two hundred and forty eight patients had an omental biopsy at the time of surgical staging for endometrial cancer (Table 1). The majority of these patients were more than 50 years old. The median age was 65 with an age range of 33-93 years. There were 187 cases of stage I (33 IA, 103 IB, 51 IC), 27 stage II, 27 stage III and seven stage IV.

Moreover, 202 (81.4\%) had endometrioid, 20 (8.1\%) serous papillary, $20(8.1 \%)$ MMMT, three $(1.2 \%)$ clear cell and three $(1.2 \%)$ sarcoma.

Overall, six cases $(2.4 \%)$ had omental involvement. Only $1 / 248(0.4 \%)$ of cases had microscopic omental deposits. Table 2 shows the clinico-pathologic features of patients with metastatic disease in the omentum. There were four cases with endometrioid type, one with serous papillary and one with MMMT. The omentum was involved in $\sim 2 \%$ of endometrioid type whereas in serous papillary and MMMT it was 5.0\%. Among those with omental metastases, 84\% were macroscopic lesions, more than half of the myometrium was involved and peritoneal washings were positive. Sixteen percent were microscopic metastases, less than half of the myometrium was involved and peritoneal washings were negative. In the endometrioid subtype, half of the cases were poorly differentiated and half were moderately differentiated.

\section{Discussion}

A discrepancy between clinical and surgical staging has been reported earlier [12]. Recent data suggested adding omentectomy as part of surgical staging. In some cases, appendicectomy has also been suggested [9]. However, the role of omental biopsy in surgical staging for endometrial cancer is unclear; although the procedure is simple and associated with minimal morbidity, it has an effect on the cost and overall length of the operation.

In this study, the risk of omental involvement is $2 \%$ in endometrioid and 5\% for non-endometrioid. In previous studies [6-10], the proportion of microscopic metastases was $25-100 \%$ of the overall omental metastases (Table 3). In our study, we found the proportion of microscopic metastases to be around $16 \%$.

Chen SS [13] found that, in the absence of gross omental lesions, microscopic omental metastases were found in
$1.5 \%$ of cases. In our study, microscopic omental metastases were $(1 / 248) 0.4 \%$.

The overall risk of omental metastasis is low $(2.4 \%)$. However, metastatic omental lesions will upstage endometrial cancer to stage IVb; post-operative radiotherapy is not indicated as the disease already spread outside the pelvis. Randomised controlled trials are needed to decide on the best management for these patients, prognosis being poor. Peritoneal washings as expected were found to be positive in most cases with omental metastases.

In half of the patients with omental disease, the adnexa were free of disease and this seems to be associated with a better prognosis.

Most omental metastases can be diagnosed by careful inspection and palpation of the omentum and the possibility of missing microscopic disease is around $0.4 \%$. Based on these figures and possible increased in morbidity and operative time, omental biopsy cannot be justified as a standard procedure in endometrial cancer staging. However, the importance of careful inspection and biopsy of suspicious lesions cannot be overemphasised.

\section{References}

1. Cancer Research UK (2005) Cancerstats: Corpus Uteri Cancer

2. Jemal A, Murray T et al (2005) Cancer Stat 55:10-30

3. Creasman WT (1990) New gynecologic cancer staging. Obstet Gynecol 75:287-288

4. Creasman WT et al (1987) Surgical pathologic spread of endometrial cancer. Cancer 60(80):2035-2041

5. FIGO stages-1988 Revision (1989) Gynecol Oncol 35:125-127

6. Saygili U, Kavas S et al (2001) Omentectomy, peritoneal biopsy and appendectomy in patients with clinical stage I endometrial carcinoma. Int J Gynecol Cancer 11(6):471-474

7. Chen SS, Spiegel G (1991) Stage I endometrial carcinoma: role of omental biopsy and omentectomy. J Reprod Med 36 (9):627-629

8. Nieto JJ, Gornall R (2002) Influence of omental biopsy on adjuvant treatment field in clinical stage I endometrial carcinoma. BJOG 109:576-578

9. Dilek S, Dilek U et al (2006) The role of omentectomy and appendectomy during the surgical staging of clinical stage I endometrial cancer. Int J Gynecol Cancer 16(2):795-798

10. Fujiwara H, Saga Y et al (2008) Omental metastasis in clinical stage I endometrioid adenocarcinoma. Int $\mathrm{J}$ Gynecol Cancer 18:165-167

11. Gehrig PA et al (2003) The role of omentectomy during the surgical staging of uterine serous carcinoma. Int J Gynecol Cancer 13:212-215

12. Ayhan A, Yarali $\mathrm{H}$ et al (1990) Comparison of clinical and surgico-pathologic staging in patients with endometrial adenocarcinoma. J Surg Oncol 43:33-35

13. Chen SS (1995) Extrauterine spread in endometrial carcinoma clinically confined to uterus. Gynecol Oncol 21:23-31 